\title{
The Influence of Chinese and Western Thinking Patterns on Translation Teaching
}

\author{
Jingjing Xie \\ College of Foreign Languages and Literature, Wuhan Donghu University, Hubei, China
}

Keywords: influence; Chinese and Western thinking patterns; translation teaching

\begin{abstract}
Chinese and English occupy two large languages in the world. The collision occurs in Chinese and Western thinking patterns, which greatly influences understanding and translation of two languages. This paper firstly introduces the relationship of language and thinking in psychology level, and indicates translation activity based on cognitive field. Then, it further explains significance of cultivating thinking ability in translation teaching. It prominently discusses the influence of Chinese and Western thinking patterns on translation teaching in four aspects. Lastly, conclusion is made. This paper aims at exploring the excellent and effective solutions to translation teaching by distinguishing between Chinese and Western thinking patterns.
\end{abstract}

\section{Introduction}

Thinking belongs to ideology in philosophy and psychology level. Gao Mingkai, famous Chinese linguist, defines that thinking is the highest form of human realization with generalized and rational attributes. Thinking related to language is not image thinking, but abstract thought. Thinking in the human brain centrally and indirectly reflects substances in nature. Without social class, thinking is a process of means and form for cognition. The well-known psychologist of the former Soviet Union, Lev Semenovich Vygotsky, founded the famous culture-history theory whose core lies in high psychological function. He studied thinking patterns and variations that children underwent in their different stage of language learning. Thus, he finally deduced from his study on child psychology that adults would follow the same rule of psychological changes when acquiring language. It shows a close relationship between thinking and language development.

Translation is a complicated process, involving language, culture and knowledge in a certain field. From the angle of translators, if language symbols as codes, they first decode original text in mental process, and then encode target language by skills and underlying information. According to Richards et al., to categorize the collected data in mind is a research method similar to coding. Informationization of language embodies thinking patterns. For translation teaching, the corresponding between two different languages seems in lexical hierarchy superficially. But what translation concerns about and translation teachers have to cultivate is thinking pattern. This thinking pattern helps understand the deep structure of the language and information in specific formation. Chinese and English are two important languages. The Chinese nation has long history and abundant culture. Its wisdom covers Chinese linguistics, philosophy, religion, arts and etc. However, English is the largest language around the world. Western countries as heads of the world's largest camp have developed advanced ideas. Their system of law, philosophy, religion, science and technology ranks the first in the world. Thus, different thinking patterns these two languages reflect are worth studying for scholars, especially for translation teaching.

\section{The Importance of Cultivating Thinking Ability in Translation Teaching}

Based on traditional viewpoints, Sapir-Whorf hypothesis discussed the relationship of language, thought, and reality. He posed the language relativity theory. Language form restricts thinking pattern. The structure of native language affects how we think of the realistic world. The family of languages varies from the eastern to western. Language makes a sharp distinction between Chinese and Westerners' ways of understanding the world. Nevertheless, Vygotsky came to the conclusion 
that thinking had appeared before language origined, which stemmed from his research on formation of language in early ages of apes and children. He thought human's experience and cognitive level prompts language develpment. As a matter of fact, it is thinking pattern that finally defines the language structure.

The fierce controversy over the determinant role in language or thinking pattern exists for scholars. But it is not ignored that language is inseparable from thinking pattern. In cognitive level, the system of understanding and analyzing the material world is affected by many factors, such as culture and nation. Language is the overt symbol, while thinking patterns is more fixed and conventional in society. The change of language in terms of times could be caught quickly. The internal mechanism of language involves structure, meaning and application, which needs the participation of thinking and creation. In translation teaching, a certain amount of translators' activeness needs to be emphasized. Teachers should encourage students to carry out translation practice based on cognition so that students can improve the effects of translation either in deep understanding or in accurate expression.

The Chinese and Western culture differ in many aspects. For China, "the Nation of rites", the thoughts of Confucianism and Daoism have greatly influenced on Chinese people. Particularly, ethics and moral values in Confucianism represented by Confucius have seized the center of social standard since ancient China. To obey rules and follow tradition is deep-rooted in their minds. This is reflected in their thinking patterns. For instance, Chinese expression ways are more implicit on the ground of the overall situation. In the same way, language system has loaded cultural factors. Chinese characters evolve from hieroglyphics which visually record people's living conditions and thoughts. Most of Chinese characters are ideographic and complex in word formation.

Western countries, especially British and American countries, have developed economically under capitalism for many years. The political system is multi-party democracy that determines holding power in turn. British and American countries had been the governors during long-term colonial period, and mostly have a sense of personal and national superiority inherently. Westerners are extroverted and keen on freedom and adventure. Their thinking patterns like analytics and legalism demonstrate capitalistically rigorous and philosophically rational characteristics. English completely reflects western thinking patterns. Unlike Chinese, English is phonographic. This kind of language is more apt to express thoughts logically and structurally. According to westerners' personality, their language is more direct and expression more explicit.

To enhance cross-cultural sensitivity is helpful for understanding and applying two languages in the process of translation. In translation teaching, the choice of translation strategies should take cultural factors into consideration. Teachers should help students improve Chinese and Western culture identification, an effective way to cultivate their thinking patterns.

\section{The Influence of Chinese and Western Thinking Patterns on Translation Teaching}

Chinese characters are ideographic. Understanding and reflection in inner heart constitute the system of perceiving outside world. Chinese thinks highly of Parataxis. Thus Chinese has more omission while it emphasizes the thought and connotation rather than form. Especially in Classical Chinese and Poetry, refined and informative language always leaves endless aftertaste. Parataxis, an important feature of Chinese language, contains wisdom accumulation of Chinese nation since ancient times.

Westerners pay attention to practice and rigorous logic, so they are more prominent both in language structure and thinking pattern. Westerners maintain rational attitude. Thus, English puts emphasis on rigid structure, which conforms to western thinking pattern. The internal relationship of utterance more relies on explicit features on lexicon, sentence structure and semantic relation. Apart from contents, form is a key point to be evaluated. Western straightforward manner and outgoing personality facilitate English externalization. Hypostaxis represents westerners' external thinking patterns.

Parataxis and hypostaxis are two common translation methods. Different thinking patterns are beneficial for understanding the formation and expression of two languages. In translation teaching, 
E-C translation could value parataxis more while C-E translation could consider hypostaxis more. In some styles, it is inclined to choose parataxis. For example, literature translation seems very perplexing, and to reconstruct the original text in an expressive manner is hard except that the underlying contents could be dug out and the translation could move and resonate with the publicity. While hypostaxis is frequently used in professional texts, such as science and technology, law, product manual, guide book and etc., for this formal style is always direct and objective. There are a great deal of technical terms which should conform to a certain norm and standard. Nevertheless, translation teachers should keep in mind that in concrete situations, parataxis and hypostaxis could be properly combined in the same text in terms of the needs of translation expression.

Chinese people appear masters in dialectical thought. The reasoning makes thought followed closely one after another. It is continuous usually in time and confined in space. From one point, thought of the event could extend in line towards direction. Linear thinking, the fixed thinking pattern has the advantage of regularity and stability. It could reach the effect of highlighting the subject and saving the analytical time. As a static thinking, linear thinking simplifies the problem and uses monism to draw black - and - white conclusion. Linear thinking makes deduction clearly and simply in one way.

Divergent thinking is not traditional thinking pattern. It is also called brainstorm, which emphasizes the way you see the world and you solve problems from diverse analysis. This thinking pattern jumps out of the bonds of convention, and gives the rein to one's imagination and activeness. American psychologist Guilford poses four features of divergent thinking: fluent, flexible, unique, and precise. Divergent thinking is such a dynamic process often used in English, for western intellectual vitality, outgoing personality, and prevailing nation adventurism.

Linear thinking and divergent thinking are unique for Chinese and Western people separately. In translation teaching, teachers should attach importance to cultivating students' divergent thinking. To understand divergent thinking of English from various perspectives is important for E-C translation. According to dynamic features of English, it exhibits variably choice both of word and sentence pattern in C-E translation. Creativity and bounding thinking should be advocated in translation teaching. Translator' subjective consciousness needs to be further enhanced in translation process. Think actively and innovate boldly. Translation activity should be logical and rigorous. Frequent mental stimulation for students is the key of translation teaching.

In 1920s and 1930s, one representative of functional grammar, Prague School considered the communicative function of language in different context. Language reflecting thinking activity embodies semantic textual and interpersonal rules, while the generation of utterance also comes into the domain of psychology, cognition and etc. Prague School, puts forward theme and rheme of the sentence, which belongs to grammar- semanteme concept. Mathesius, the founder of Prague School, proposes actual division of the sentence. Theme gives known information, while new information and focus are put into rheme. Each sentence has its own thematic structure according to the information distribution and grammar structure arrangement.

Thematic progression involves thematic cohesion in the whole text. The interrelationship and integration shows the whole text elaborately and reasonably. Therefore, all relations between themes and rhemes in text are considered as thematic progression. It is thematic progression that ensures this step-by-step approach to inducing and deepening the central meaning of the text logically. The basic pattern of theme alterations in text is described as thematic progression. Huang Guowen sums up six patterns of thematic progression: parallel progression, constant progression, linear progression, split progression, alternating progression and crossing progression.

Chinese is a topic-prominent language. Although topic is not equivalent to theme, topic-comment construction of Chinese is actually apt to be understood. For Chinese, its synthetic characteristic is more obvious so as to arrange and distribute semanteme of the text closely and harmoniously. In translation teaching, it is important to instruct students to distinguish between theme and actual subject, and turn this kind of Chinese structure into subject-predicate structure in order to understand and translate them properly and accurately. As a subject-prominent analytic language, English attaches importance to logical orders and structure of the discourse. In this way, 
to grasp its internal semantic relationship seems more important for translation teaching. In brief, it is indispensable that thematic progression pattern reflecting different thinking pattern functions in textual analysis for translation teaching.

Harris puts forward discourse analysis in his book Discourse Analysis in 1952. The discourse or text, not fragment of utterance, can be studied both from structure and function perspectives. In 1981, Beaugrande and Dressler described and explained the discourse formation from interpersonal angle. They gave seven communicative occurrences satisfied with textuality: cohesion, coherence, intentionality, acceptability, informationlity, situationlity, and intertextulity. Among them, cohesion is the connective way that ensures the presentation of fluent and natural text. Different sentences are correlated in the text, so cohesion involves how to reasonably integrate them.

Textual cohesion is both external and internal. In form, it is visible for external cohesion such as ellipsis, reference, conjunction and substitution. However, internal cohesion is implicit so that only with the underlying semantic and textual relations of the text could we properly grasp the textual connotation. In translation teaching, teachers should remind students that Chinese and English show different characteristics of textual cohesion in terms of their different thinking patterns.

Chinese is synthetic whereas English is analytic. Now that Chinese people are good at dialectic thinking, translation teachers should encourage students to analyze the internal complicated semantic relations of Chinese in C-E translation. English, an analytic language, attaches importance to structure and external cohesion, hence, in translation teaching, teacher should enhance students' sensitivity of English explicit cohesion, so properly adding relatives, personal references and cohesive marks to translation is very frequent in C-E translation.

In E-C translation, teachers should instruct students to figure out obscure and hidden semantic relations through English explicit marks. While in C-E translation, it is necessary to simplify some obvious marks presenting external cohesion and increase the usage frequency of substantives and make concrete semantic relations clearly in accordance with Chinese context.

\section{Conclusion}

The relationship of thinking and language belongs to Cognitive Linguistics. Language is the special form which can reflect thinking, while thinking can represent and promote language. Thus, different thinking pattern Chinese and English culture and their languages demonstrates is worth studying, especially for translation, a complex activity of encoding and decoding. In translation teaching, cultivating thinking ability becomes more important. On one hand, mechanism of language in cognitive level involves many factors. The system of understanding and analyzing the material world is so complex. Translators' activeness is the key for successful translation. On the other hand, teachers should help students improve Chinese and Western culture identification ability, which is beneficial for which kind of translation strategy they choose.

Traditional translation teaching lays emphasis on translation theory and practice, but thinking pattern which confuses plenty of students usually isn't valued. It is innovative to combine different thinking pattern with translation teaching. Chinese and Western thinking patterns have great influence on translation teaching in four aspects: parataxis and hypostaxis, linear thinking and divergent thinking, thematic progression pattern and textual cohesion way. Chinese and English are two languages of distinctive characteristics. In most cases, Chinese and Western thinking patterns determine the formation and expression of translation. In translation teaching, teachers should enhance the contrastive training of Chinese and Western thinking patterns in order to help students understand and translate text accurately and intelligently.

\section{References}

[1] Gao M. K. (1956). Language and Thinking. Beijing: SDX Joint Publishing Company.

[2] Li G. M. (2016). Language and Translation Practice. Chengdu: Sichuan University Press.

[3] Sonia C. (2009). Translation Teaching. Shanghai: Shanghai Foreign Language Education Press. 